\title{
NME4 may enhance non-small cell lung cancer progression by overcoming cell cycle arrest and promoting cellular proliferation
}

\author{
WENQIAN WANG ${ }^{1,2^{*}}$, MING DONG $^{3 *}$, JIE CUI $^{1,2}$, FEI XU ${ }^{1,2}$, CHEN YAN $^{1,2}$, CHENG MA $^{1,2}$, \\ LA YI $^{1,2}$, WEIFENG TANG ${ }^{1,2}$, JINGCHENG DONG $^{1,2}$ and YING WEI ${ }^{1,2}$ \\ ${ }^{1}$ Department of Integrative Medicine, Huashan Hospital, Fudan University; \\ ${ }^{2}$ The Institutes of Integrative Medicine of Fudan University, Shanghai 200040; \\ ${ }^{3}$ Gumei Community Health Center of Minhang District of Shanghai, Shanghai 201102, P.R. China
}

Received July 11, 2018; Accepted May 28, 2019

DOI: $10.3892 / \mathrm{mmr} .2019 .10413$

\begin{abstract}
Nucleoside diphosphate kinase 4 (NME4) is abnormally expressed in a variety of cancer types. However, the function of the NME4 gene in non-small cell lung cancer (NSCLC) remains to be elucidated. In order to investigate the role of NME4 in NSCLC, the present study detected the expression of the NME4 gene in the Cancer Genome Atlas database, and in BEAS-2B, NCI-H1299 and A549 cell lines. NME4 was significantly overexpressed in NSCLC tissues and NSCLC cell lines. Furthermore, lentivirus-mediated knockdown vector infection, cell proliferation, cell cycle, apoptosis, colony formation and MTT assays were conducted to explore the effect of NME4 on NSCLC in vitro. After knockdown of NME4 with short hairpin RNA, the cell cycle was arrest at the G1 phase, and proliferation and colony formation were inhibited in the NCI-H1299 and A549 cell lines. The present results suggested that NME4 may serve as a novel tumor promoter, capable of enhancing NSCLC progression by overcoming cell cycle arrest and promoting proliferation.
\end{abstract}

\section{Introduction}

Lung cancer is one of the most common types of cancer and the leading cause of cancer-associated mortality in America (1). Of all lung cancer cases, 83\% are of the non-small cell lung cancer (NSCLC) histological type (2). Despite advances in lung cancer treatment (3), NSCLC

Correspondence to: Dr Ying Wei or Dr Jingcheng Dong, Department of Integrative Medicine, Huashan Hospital, Fudan University, 12 Middle Urumqi Road, Shanghai 200040, P.R. China E-mail: weiying_acup@126.com

E-mail: jcdong2004@126.com

*Contributed equally

Key words: nucleoside diphosphate kinase 4, non-small cell lung cancer, cell proliferation, apoptosis, cell cycle remains an aggressive type of lung cancer due to its metastatic potential (3). Considering the important contribution of NSCLC to the worldwide burden of cancer (4), it is important to further elucidate the mechanisms and to explore novel treatments.

Nucleoside diphosphate kinase 4 (NME4), also known as non-metastatic clone 23 human isoform 4 (NM23-H4), is one of the members of the NM23 family (5). The NME4 protein, which contains a mitochondrial target sequence (6), has been shown to be associated with mitophagy (7), cell apoptosis (8), invasive potential (9) and inflammatory reactions (10). Kagan et al (7) found that high expression of wild-type NME4 in HeLa cells increases cardiolipin externalization, thus regulating the elimination of mitochondria via autophagy. In most cancer models, the loss of autophagy reduces tumor growth, survival and proliferation (11). Genomic aberrations or altered gene expression have been observed with respect to NME4 in several types of cancer, such as gastric cancer $(12,13)$, colorectal carcinoma (14), renal tumors (14), breast cancer (15), testicular germ cell tumors (16) and large cell anaplastic lymphoma (17). However, rarely has the relationship between NME4 and NSCLC been reported. A previous study demonstrated that a decreased $n-6 / n-3$ fatty acid ratio reduces the invasive potential of human lung cancer cells by downregulating the expression of cell adhesion/invasion-associated molecules such as NME4 (9). In addition, autophagy in NSCLC preserves mitochondrial quality and regulates their abundance to promote tumorigenesis (18). The NME4 protein is involved in the function of the outer and inner mitochondrial membranes (19) and is critical for mitochondrial mitophagy (10). This indicates that the NME4 gene may be implicated in the mechanisms of NSCLC progression.

The present study was designed to further explore the effect of NME4 on NSCLC in vitro. Firstly, The Cancer Genome Atlas (TCGA) database and reverse transcription-quantitative PCR (RT-qPCR) were used to assess the expression of NME4 in NSCLC tissues and NSCLC cell lines. Then, short hairpin RNA (shRNA) was used to silence the expression of NME4 in NSCLC cell lines; cell proliferation, cell cycle, apoptosis, colony formation and MTT assays were performed to clarify the possible role of NME4. 


\section{Materials and methods}

Analysis of NME4 in NSCLC from TCGA. TCGA (http://cancergenome.nih.gov/) is a collection of microRNA-sequencing (seq), RNA-seq, single-nucleotide polymorphism array, DNA methylation and exome sequencing data, among other data types. TCGA can be used to analyze complex cancer genomics and clinical parameters. In the present study, data of RNA-Seq in lung adenocarcinoma for NME4 were extracted from TCGA, and the expression levels of NME4 in each case was calculated according to the distributions of the exon reads.

Cell lines and cell culture. The NCI-H1299 and A549 cell lines, which are commonly used in NSCLC research, were purchased from the Cell Bank of the Shanghai Institute of Cell Biology, Chinese Academy of Sciences. Cells were cultured in RPMI-1640 medium (Invitrogen; Thermo Fisher Scientific, Inc.) containing 10\% FBS (Invitrogen; Thermo Fisher Scientific, Inc.), $100 \mathrm{U} / \mathrm{ml}$ penicillin, $100 \mathrm{mg} / \mathrm{ml}$ streptomycin and $2 \mathrm{mmol} / 1 \mathrm{~L}$-glutamine at $37^{\circ} \mathrm{C}$ in humidified air containing $5 \% \mathrm{CO}_{2}$. BEAS-2B cells were cultured in DMEM/F-12 (Gibco; Thermo Fisher Scientific, Inc.), containing 5\% FBS (Invitrogen; Thermo Fisher Scientific, Inc.) and $100 \mathrm{U} / \mathrm{ml}$ penicillin, $100 \mathrm{mg} / \mathrm{ml}$ streptomycin and $2 \mathrm{mmol} / \mathrm{l} \mathrm{L}$-glutamine at $37^{\circ} \mathrm{C}$ in humidified air containing $5 \% \mathrm{CO}_{2}$. The complete medium in the present study was changed at least once every 2 days.

$R N A$ extraction, reverse transcription $(R T)$ and quantitative $P C R(q P C R)$. TRIzol ${ }^{\circledR}$ reagent (Invitrogen; Thermo Fisher Scientific, Inc.) and M-MLV Reverse Transcriptase (Promega Corporation) were used to extract the total RNA from cells and to perform RT, respectively. RT was conducted at $73.5^{\circ} \mathrm{C}$ for $7 \mathrm{~min}$, in an ice water mixture for $5 \mathrm{~min}$, at $43.5^{\circ} \mathrm{C}$ for $1 \mathrm{~h}$ and finally at $73.5^{\circ} \mathrm{C}$ for $3 \mathrm{~min}$. Next, qPCR was performed on a MX3000P qPCR System (Agilent Technologies, Inc.) using the Takara SYBR Master Mixture (Takara Biotechnology Co., Ltd.). NME4 expression was quantified in real time with SYBR Green and normalized to GAPDH expression, which was used as an internal control. The denaturing, annealing and extension conditions of each PCR cycle were $95^{\circ} \mathrm{C}$ for $30 \mathrm{sec}, 95^{\circ} \mathrm{C}$ for $5 \mathrm{sec}$ and $60^{\circ} \mathrm{C}$ for $30 \mathrm{sec}$, respectively. The gene-specific primer pairs were as follows: NME4 forward, 5'AGGGTACAATGTCGTCCGC3' and reverse, 5'GACGCT GAAGTCACCCCTTAT3'; and GAPDH forward, 5'TGACTT CAACAGCGACACCCA3' and reverse, 5'CACCCTGTTGCT GTAGCCAAA3'. Each experiment was repeated twice in triplicate. The expression of target genes was calculated using the $2^{-\Delta \Delta C q} \operatorname{method}(20)$.

Lentivirus-mediated knockdown vector transduction. Lentiviral vectors for NME4-shRNA, which were purchased from Shanghai GeneChem Co., Ltd., were used to examine the function of NME4 (human NME4 cDNA; National Center for Biotechnology Information accession no. NM_005009); the vector used was hU6-MCS-CMV-EGFP. A total of two experimental groups for each cell line were constructed. The shNME4 group was infected with NME4-shRNA lentivirus (5'TGATTGGACACACCGACTC3'), while control cells were infected with a lentivirus containing a scramble sequence (5'TTCTCCGAACGT GTCACGT3'). NCI-H1299, A549 and BEAS-2B cells in 6-well plates $\left(2 \times 10^{6}\right.$ cells/well) were infected with lentiviral particles at a multiplicity of infection of $10\left(5 \times 10^{6} \mathrm{TU} / \mathrm{ml}\right)$ for $16 \mathrm{~h}$ using polybrene (Sigma-Aldrich; Merck KGaA), following which the medium was replaced with fresh culture medium and cells were cultured for a further $56 \mathrm{~h}$; the knockdown efficiency was detected by RT-qPCR. Prior to transduction, the medium of A549 cells was replaced with Opti-Minimal Essential Medium (Gibco; Thermo Fisher Scientific, Inc.) + polybrene, whereas the H1299 cell medium was replaced with RPMI-1640 medium + polybrene; BEAS-2B cells were cultured with DMEM/F12 + polybrene for transduction.

Cell proliferation. NME4-shRNA-transfected cells and control cells were collected with $0.25 \%$ trypsin-EDTA and resuspended in RPMI-1640 standard medium once they had achieved logarithmic growth. Cells were then seeded at a density of 2,000 cells/well and further incubated at $37^{\circ} \mathrm{C}$ in humidified air containing $5 \% \mathrm{CO}_{2}$ for 5 days. GFP expression (from the hU6-MCS-CMV-EGFP vector) was measured in each well using a Cellomics ArrayScan VT1 (Thermo Fisher Scientific, Inc.) for a 5-day period. Data were mapped, and cell proliferation curves were generated for each group.

Cell cycle analysis. A total of $2 \times 10^{6}$ cells were seeded in 6-well plates, incubated overnight and then starved in culture medium without serum for $12 \mathrm{~h}$ to synchronize their cell cycle. Next, cells were cultured in complete medium for $48 \mathrm{~h}$, harvested, washed and fixed in $70 \%$ ethanol overnight at $4^{\circ} \mathrm{C}$. Cells were then washed, and stained with propidium iodide (PI; $10 \mu \mathrm{g} / \mathrm{ml})$ and RNase A (100 $\mu \mathrm{g} / \mathrm{ml})$ at room temperature for $30 \mathrm{~min}$, followed by flow cytometry detection using a Guava easyCyte HT flow cytometer (EMD Millipore) and Shortcut 3.1 software (Incyte Corporation). Cells with sub-G1 DNA content were considered apoptotic cells. All experiments were performed in triplicate.

Apoptosis analysis. The cells in each group were harvested with $0.25 \%$ trypsin, washed once with ice-cold PBS and analyzed with the Annexin V-Allophycocyanin Apoptosis Detection kit with PI (eBioscience; Thermo Fisher Scientific, Inc.) to assess apoptosis. Cells were centrifuged at $200 \mathrm{x} \mathrm{g}$ and room temperature for 10-15 min, resuspended and incubated at room temperature for $10-15 \mathrm{~min}$ according to the manufacturers' instructions, followed by flow cytometry detection of the apoptotic cells using Shortcut software. Detection was performed at $72 \mathrm{~h}$ after NME4 knockdown.

Colony formation assay. Cell colony formation was examined by a colony formation assay. Cells in each group were digested with $0.25 \%$ trypsin and seeded into 6-well plates at a density of 800 cells/well. After 2 weeks of incubation, colonies that included $>50$ cells were scored as surviving colonies. Colonies were visualized under a fluorescence microscope (IX71; Olympus Corporation). Cells were washed with PBS, fixed with $4 \%$ paraformaldehyde (Sangon Biotech Co., Ltd.) for $30 \mathrm{~min}$ at room temperature and stained with $500 \mu \mathrm{l}$ Giemsa solution (ECM550; Chemicon International; Thermo Fisher 
A

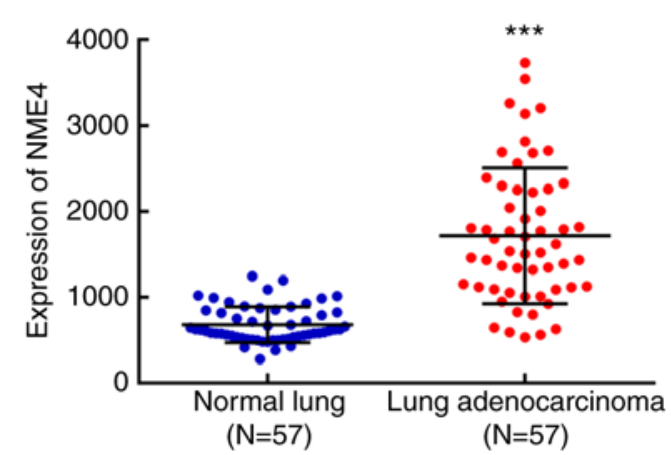

C

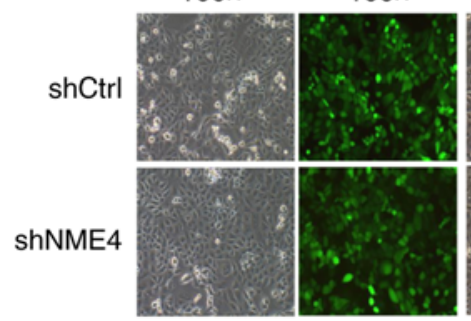

$\mathrm{NCl}-\mathrm{H} 1299$

Brightfield Fluoroscope Brightfield Fluoroscope 100x 100x

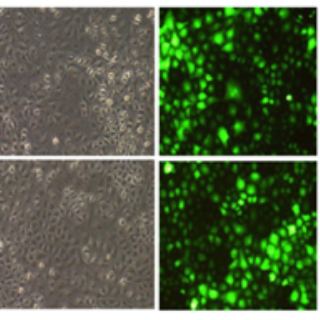

A549
B

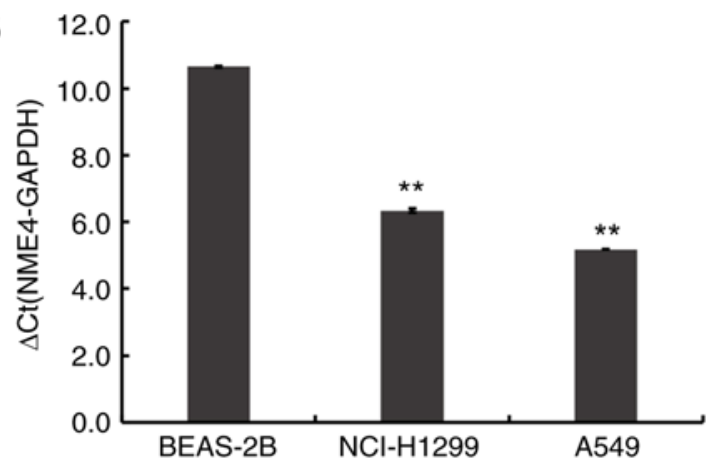

D

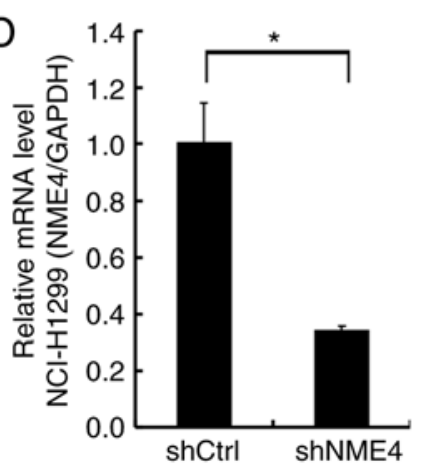

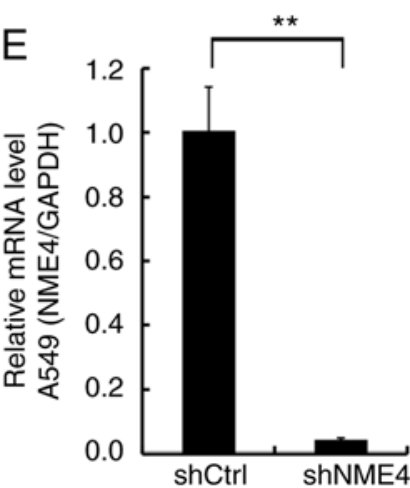

Figure 1. Results for NME4 expression in TCGA database and different cell lines. (A) NME4 was highly expressed in lung adenocarcinoma compared with non-cancerous lung tissues, based on TCGA database. ${ }^{* * *} \mathrm{P}<0.001$ vs. normal lung. (B) Expression levels of NME4 mRNA in BEAS-2B, NCI-H1299 and A549 cell lines $(\mathrm{n}=3) .{ }^{* *} \mathrm{P}<0.01$ vs. BEAS-2B. (C) After lentiviral transduction for $72 \mathrm{~h}$, the infection rate of cells reached $>80 \%$ and the status of the target cells was normal. (D) After lentiviral transduction, relative NME4 mRNA expression was significantly inhibited in the NCI-H1299 NME4-shRNA silenced cells (shNME4 group) compared with the negative control cells, as assessed by RT-qPCR. (E) After lentiviral transduction, relative NME4 mRNA expression was significantly inhibited in the A549 NME4-shRNA silenced cells compared with the negative control cells, as assessed by RT-qPCR. GAPDH was used as an internal control. Comparisons between two groups were performed by Student's t-test and between multiple groups by ANOVA. Error bars indicate SD. "P<0.05, ${ }^{* *}$ P<0.01. RT-qPCR, reverse transcription-quantitative PCR; NME4, nucleoside diphosphate kinase 4; sh, short hairpin; TCGA, The Cancer Genome Atlas.

Scientific, Inc.) for $20 \mathrm{~min}$ at room temperature. Following several washes with deionized distilled water, the cells were allowed to air dry at room temperature. Colonies were counted and images were captured with a digital camera under light microscopy (magnification, x100; XDS-100; Shanghai CaiKang Optical Instrument Co., Ltd.). The assay was repeated three times.

MTT assay. Cell proliferation was examined by MTT assay. Cells $(2,000$ cells $/ \mathrm{ml})$ were seeded into 96 -well plates and incubated at $37^{\circ} \mathrm{C}$ for $24,48,72,96$ or $120 \mathrm{~h}$. At $4 \mathrm{~h}$ prior to each time point, $0.5 \%$ MTT solution (Thermo Fisher Scientific, Inc.) was added, followed by incubation for $4 \mathrm{~h}$ at $37^{\circ} \mathrm{C}$. The cell supernatants were discarded, and the formazan crystals were dissolved in $100 \mu 1$ dimethyl sulfoxide. The optical density (OD) of each group was measured using a microplate reader (M2009PR; Tecan Group, Ltd.) at a wavelength of $490 / 570 \mathrm{~nm}$.

Statistical analysis. Data were analyzed using SPSS 19.0 statistical software (IBM Corp.). Data are expressed as the mean $\pm \mathrm{SD}$ of three experimental repeats. Comparisons between two groups were performed by Student's t-test and between multiple groups were performed by one-way ANOVA followed by Student-Newman-Keuls post hoc test. $\mathrm{P}<0.05$ was considered to indicate a statistically significant difference.

\section{Results}

Information from TCGA database. To further elucidate the relationship between NME4 and NSCLC, a clinical study was performed using original data from TCGA. It was found that NME4 was highly expressed in lung adenocarcinoma compared to non-cancerous lung tissues ( $\mathrm{P}<0.001$; Fig. 1A).

NME4 is overexpressed in NSCLC cell lines and silenced upon lentivirus transduction. To investigate the NME4 mRNA expression levels in NSCLC, RT-qPCR was performed in BEAS-2B, NCI-H1299, and A549 cells. NME4 mRNA expression levels were high in NCI-H1299 and A549 cells compared to BEAS-2B (Fig. 1B). Then, NCI-H1299 and A549 cells were transfected with a NME4-shRNA lentivirus or a scramble vector lentivirus. Upon lentiviral infection for $72 \mathrm{~h}$, the infection rate was $>80 \%$ (Fig. 1C), making these cells suitable for subsequent experiments. Upon NME4-shRNA lentiviral transduction, RT-qPCR analysis revealed that NME4-shRNA decreased the mRNA expression of endogenous NME4 significantly ( $\mathrm{P}<0.05$; Fig. 1D and E).

NME4 silencing inhibits NCI-H1299 and A549 cell proliferation. Following lentiviral transduction, the proliferation of NCI-H1299 and A549 cells was obviously inhibited in NME4-shRNA-silenced cells compared with control cells, as shown by GFP-based Cellomics ArrayScan 
A
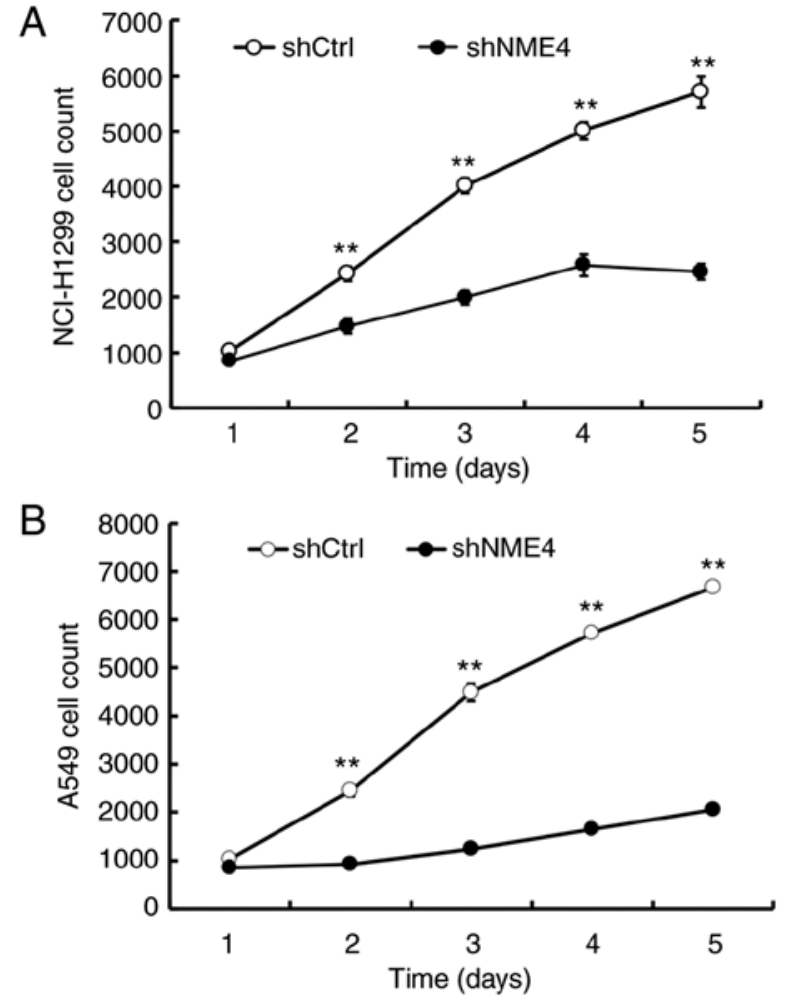
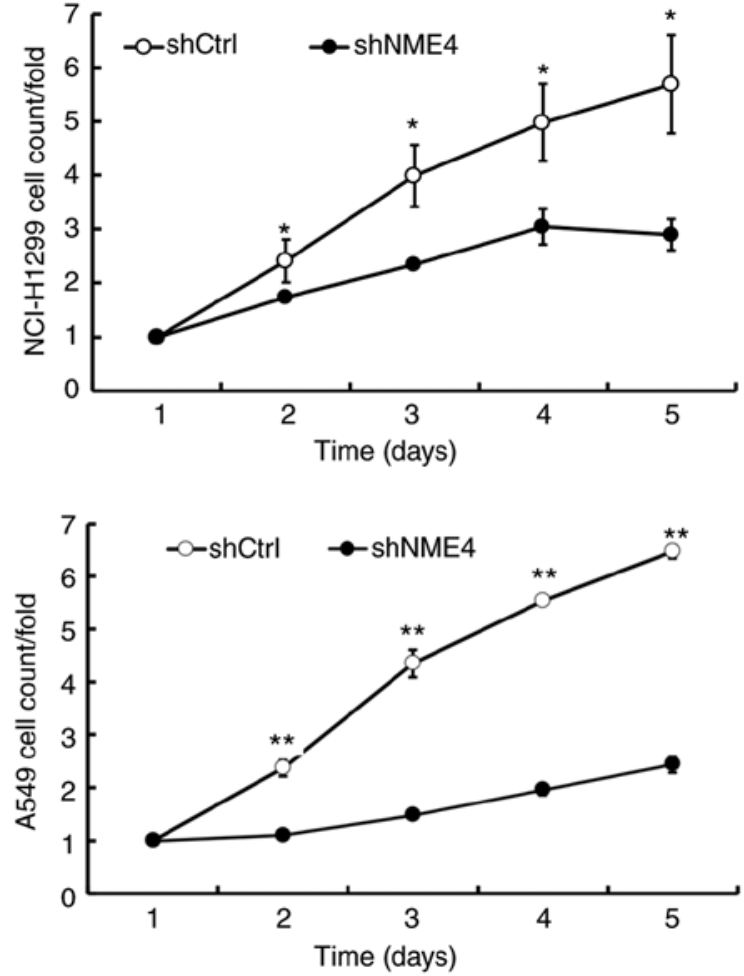

Figure 2. Cell proliferation analysis by green fluorescent protein-based imaging. (A) After lentiviral transduction of NCI-H1299 cells, cell counts and fold-changes were significantly inhibited in NME4-shRNA-silenced cells compared with control cells. (B) After lentiviral transduction of A549 cells, cell counts and fold-changes were significantly inhibited in NME4-shRNA-silenced cells compared with control cells. Statistical significance was assessed by ANOVA for the comparison of multiple groups. Error bars indicate SD. ${ }^{*} \mathrm{P}<0.05,{ }^{* *} \mathrm{P}<0.01$ vs. shCtrl. NME4, nucleoside diphosphate kinase 4; sh, short hairpin; Ctrl, control.

VTI imaging (Fig. 2). The cell numbers in each group were monitored for 5 days. Cell numbers were significantly reduced in the NME4-shRNA-silenced cells (Fig. 2). These results suggested that NME4 was associated with NCI-H1299 and A549 cell proliferation.

NME4 silencing induces cell cycle progression. Cell cycle distribution was assessed to further elucidate the growth-suppressing effect of NME4-shRNA on NCI-H1299 and A549 cells. Compared with the control group, NME4-shRNA significantly increased the fraction of G1-phase cells in the NME4-shRNA group (Fig. 3). The results suggested that NME4 silencing may induce cell cycle arrest at the G1 phase, and the effect of NME4 on the cell cycle was time-dependent. G1 arrest was more pronounced in A549 cells compared with NCI-H1299 cells (Fig. 3), which may be due to the less efficient knockdown of NME4 (Fig. 1C and D). Whether the silencing of NME4 was associated with apoptosis in NCI-H1299 and A549 cells was further investigated. The proportions of apoptotic cells were increased by only $3-5 \%$ in NME4-silenced groups of NCI-H1299 and A549 cells when compared with control cells, comparable to the effects observed in shRNA-transduced BEAS-2B cells (Fig. 4). These data do not suggest that NME4 had an effect on the apoptosis of NCI-H1299 and A549 cells. This level of increase in apoptosis may be due to the state of the cells under the conditions of the assay. These results may indicate that NME4 silencing interrupted cell cycle progression and thus affected the progression of NSCLC, rather than increasing cell apoptosis.
NME4 silencing reduces NCI-H1299 and A549 cell colony formation. Silencing of NME4 suppressed the anchorage-independent growth of NCI-H1299 and A549 cells in soft agar (Fig. 5). Infection with NME4-shRNA in NCI-H1299 and A549 cells significantly reduced the number of cell clones $(\mathrm{P}<0.01)$, which confirmed that the silencing of NME4 suppressed the proliferative potential of NCI-H1299 and A549 cells, and revealed the critical role of NME4 in the survival of NSCLC cells.

MTT assay. An MTT assay was performed to detect the proliferation of NCI-H1299 and A549 cells upon transfection with NME4-shRNA for $24,48,72,96$ or $120 \mathrm{~h}$. The OD value at $490 \mathrm{~nm}$ indicated the viability of the cells. The results of MTT assay demonstrated that the silencing of NME4 significantly decreased the proliferation of NCI-H1299 and A549 cells (Fig. 6), which indicates that NME4 contributes to NSCLC proliferation.

\section{Discussion}

As one of the most common cancer types, NSCLC also has the highest mortality rate. Although the mainstays of lung cancer treatment may slow down tumor growth, the genetic versatility of tumor cells may induce resistance to the currently available therapies. Thus, it is critical to develop novel, effective and safe approaches for NSCLC treatment.

NME4 is one of the members of the NM23 family (5), which have been postulated to be involved in cell adhesion 

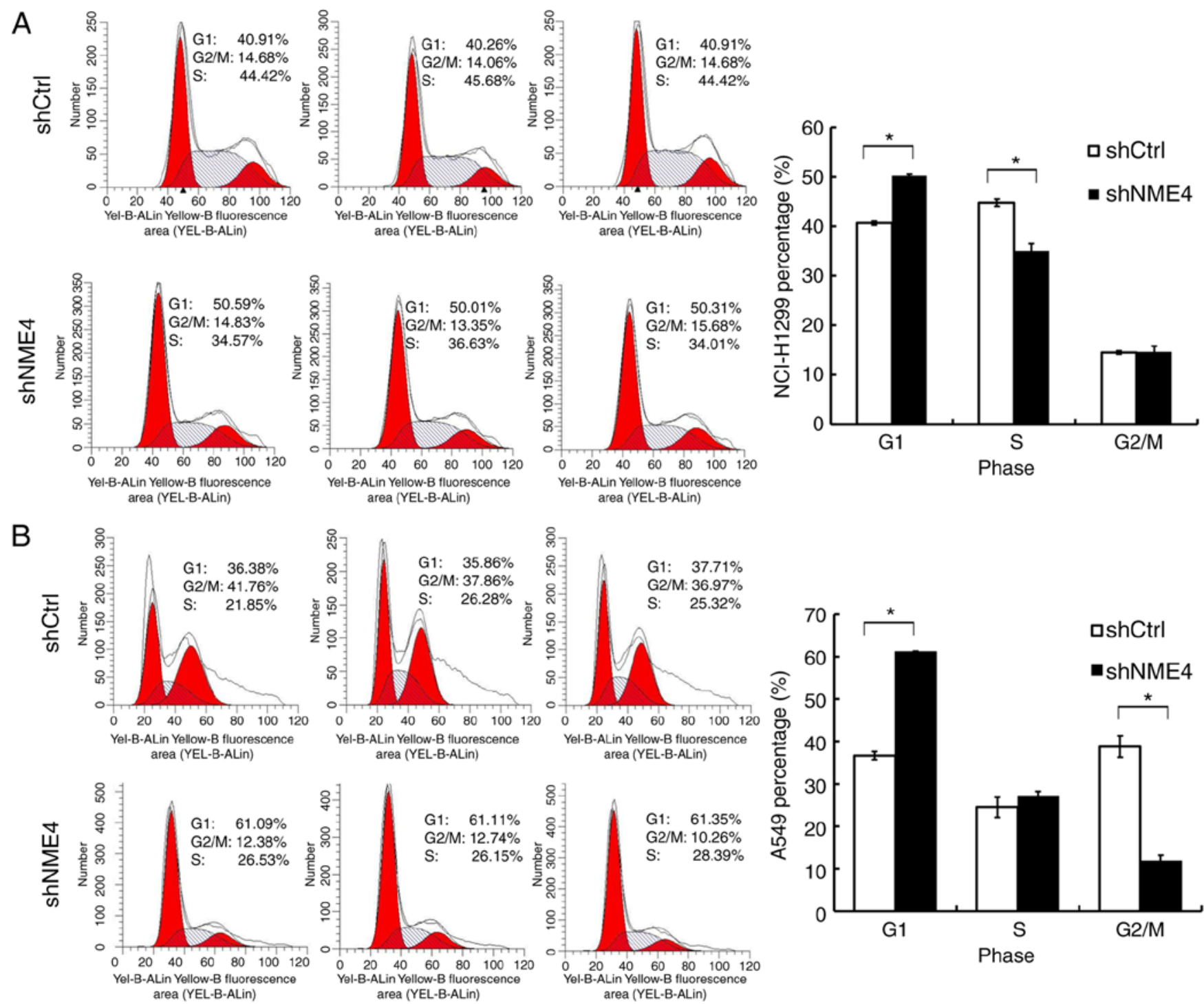

Figure 3. Effects of the silencing of NME4 on cell cycle distribution. (A) After lentiviral transduction of NCI-H1299 cells, cell cycle assessment showed that knockdown of NME4 in NCI-H1299 cells induced accumulation in the G1 phase, but a decreased number of cells in the S phase. (B) After lentiviral transduction of A549 cells, cell cycle assessment showed that knockdown of NME4 in A549 cells induced accumulation in the G1 phase, but a decreased number of cells in the G2/M phase. The percentages of cells in different phases are shown as the mean $\pm \mathrm{SD}$ of three independent experiments. Statistical significance was assessed by two-tailed Student's t-test. Error bars indicate SD. "P<0.05. NME4, nucleoside diphosphate kinase 4; sh, short hairpin; Ctrl, control.

and migration (21), as well as possessing NDPK activity and being involved in DNA repair mechanisms $(12,22)$. The NME4 protein contains a mitochondrial target sequence $(19,23,24)$, and is involved in the function of the outer and inner mitochondrial membranes (19), which is critical for mitochondrial mitophagy (7). Mitophagy can maintain the normal metabolism of cells and prevent cellular stress responses and genomic damage, thereby inhibiting tumor development. Furthermore, tumor cells enhance their tolerance to hypoxia and low nutrition by enhancing mitophagy so as to survive; mitophagy serves a significant role in promoting tumor development $(25,26)$. Cancer cells require altered mitochondrial functions, including organelle dynamics, to resist bioenergetic/biosynthetic reprogramming for supporting proliferation, migration and invasion (27). Since NME4 plays a role in all of these, it is tempting to speculate that its altered expression or function may affect the fate of a cancer cell. At the same time, it has been reported that NME4 may constitute an important link between energy metabolism and cellular regulation. Abnormal expression of the NME4 gene may induce an imbalance of nucleotide pools in the mitochondria, resulting in the failure of checkpoint controls and the accumulation of subsequent genetic alterations, thus contributing to tumorigenesis (12).

Therefore, it was hypothesized that NME4 may participate in an important mechanism in NSCLC. NME4 was significantly overexpressed in NSCLC tissues, based on TCGA data. Furthermore, the present study demonstrated that NME4 mRNA expression levels were high in human NSCLC cell lines via qPCR. The present study also established low expression of NME4 in A549 and NCI-H1299 cells using lentivirus-mediated technology. When NME4 was expressed at reduced levels, the cell proliferation rate and colony formation of A549 and NCI-H1299 cells markedly decreased. Low expression of NME4 also resulted in cell cycle arrest at the G1 phase in these NSCLC cell lines. Based on these observations, 

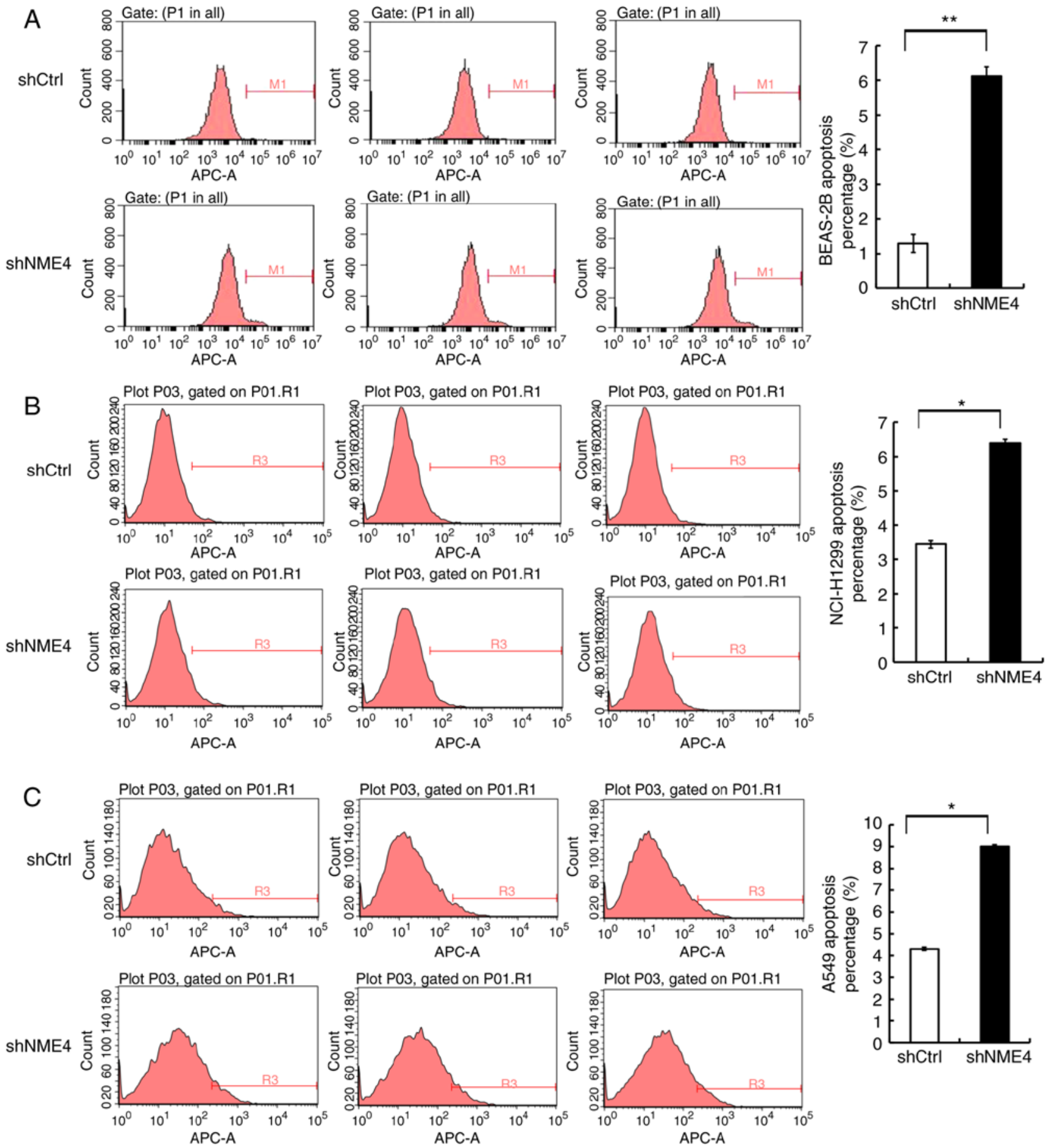

Figure 4. Effects of the silencing of NME4 on cell apoptosis. (A) After lentiviral transduction of BEAS-2B cells, apoptosis was increased in NME4-shRNAsilenced cells compared with control cells. (B) After lentiviral transduction of NCI-H1299 cells, apoptosis was increased in NME4-shRNA-silenced cells compared with control cells. (C) After lentiviral transduction of A549 cells, apoptosis was increased in NME4-shRNA-silenced cells compared with control cells. Apoptotic rates were analyzed by Annexin V-APC/propidium iodide assay. The percentages of cells in different phases are shown as the mean \pm SD of three independent experiments. Statistical significance was assessed by two-tailed Student's t-test. Error bars indicate SD. ${ }^{*} \mathrm{P}<0.05,{ }^{* * *} \mathrm{P}<0.01$. NME4, nucleoside diphosphate kinase 4; sh, short hairpin; Ctrl, control; APC, allophycocyanin.

it was concluded that NME4 may serve as a novel tumor promoter capable of enhancing NSCLC progression. To the best of our knowledge, this was the first study to identify that NME4 expression may exhibit tumor-promoting potential in NSCLC. However, some limitations exist in the present analysis. First, only two types of NSCLC cell lines were used, which may result in a loss of comprehensiveness. Second, the less efficient knockdown of NME4 in the NCI-H1299 cell line may weaken the interpretation of the role of this gene from the results of the present study.

In conclusion, the present study determined that NME4 serves critical roles in NSCLC development. Although the detailed mechanisms remain to be elucidated, the critical role of NME4 in NSCLC development may provide evidence for the development of novel therapeutics against NME4 for the treatment of NSCLC. 
A
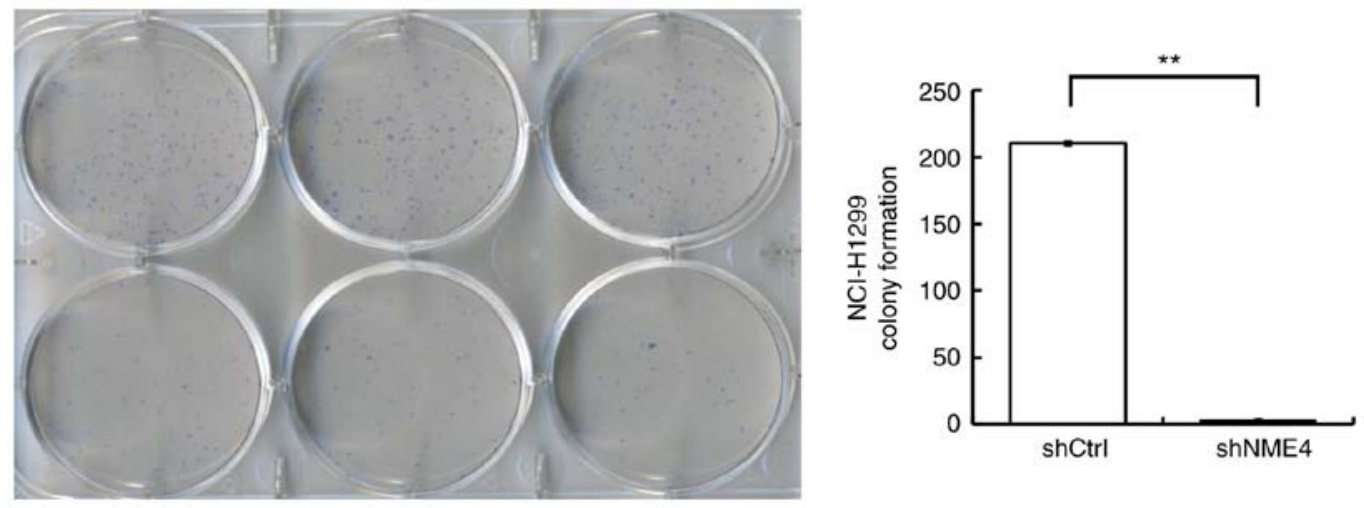

B
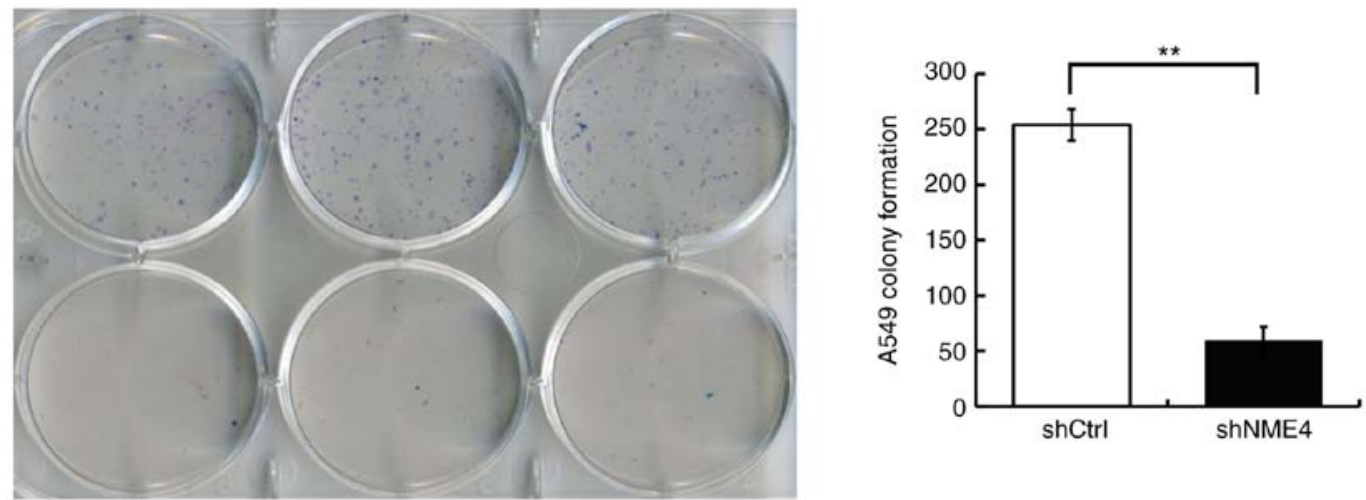

Figure 5. Effects of the silencing of NME4 on cell colony formation. (A) After lentiviral transduction of NCI-H1299 cells, the NME4-shRNA-silenced cells displayed a significantly reduced number of cell colonies compared with control cells. (B) After lentiviral transduction of A549 cells, the NME4-shRNA-silenced cells displayed a significantly reduced number of cell colonies compared to the control cells. Whole-plate fields were photographed and are presented. The number of cell colonies from triplicate counts in a representative experiment are presented. Statistical significance was assessed by two-tailed Student's t-test. Error bars indicate SD. ${ }^{* *} \mathrm{P}<0.01$. NME4, nucleoside diphosphate kinase 4; sh, short hairpin; Ctrl, control.
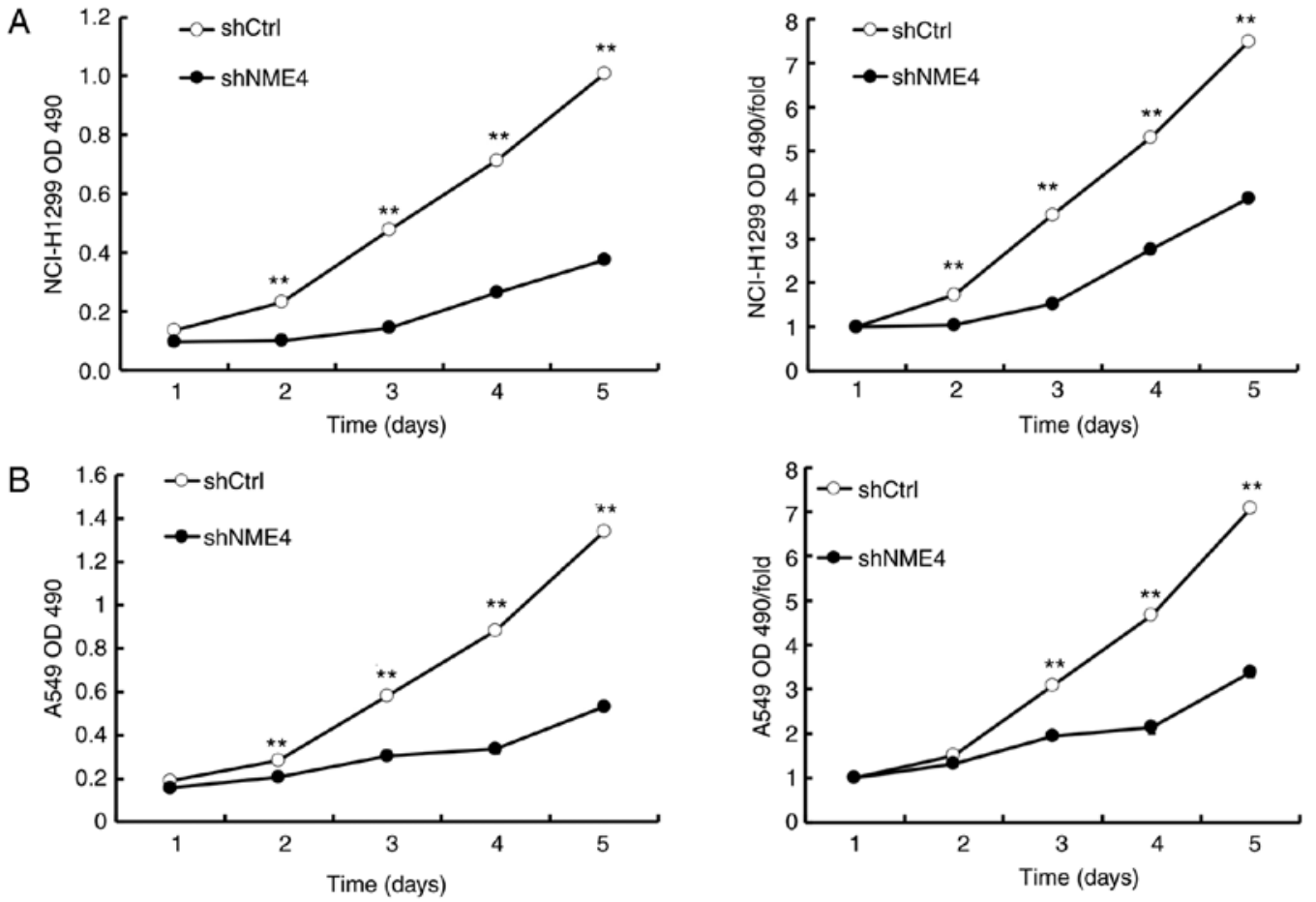

Figure 6. Cell proliferation analysis by MTT assay. (A) After lentiviral transduction of NCI-H1299 cells, MTT assays were performed on the indicated days to show the proliferation of NCI-H1299 cells. The OD values and fold-changes were significantly reduced in the NME4-shRNA-silenced cells as compared to the control cells. (B) After lentiviral transduction of A549 cells, the OD values and fold-changes were significantly reduced in the NME4-shRNA-silenced cells as compared to the control cells. Statistical significance was assessed by ANOVA for the comparison of multiple groups. Error bars indicate SD. ${ }^{* *} \mathrm{P}<0.01$ vs. shCtrl. NME4, nucleoside diphosphate kinase 4; sh, short hairpin; Ctrl, control; OD, optical density. 


\section{Acknowledgements}

Not applicable.

\section{Funding}

This work was supported by the National Natural Science Foundation of China (grant nos. 81403476, 81704154 and 81573758), Development Project of Shanghai Peak Disciplines-Integrated Chinese and Western Medicine, Shanghai Health and Family Planning Commission Program for Traditional Chinese Medicine (grant no. 2016JP001), Young Elite Scientists Sponsorship Program by China Association for Science and Technology (grant no. 2018QNRC001) and the Scientific Research Project of Shanghai Science and Technology commission (grant no. 17401930300).

\section{Availability of data and materials}

The datasets used and/or analyzed during the current study are available from the corresponding author on reasonable request.

\section{Authors' contributions}

YW and JD conceived and designed the study. WW and MD performed the majority of the experiments, performed preliminary analysis and drafted the manuscript. YW reviewed the data analysis and revised the manuscript. JC, FX, CY, CM, LY and WT participated in the completion of the experiments. All the authors read and approved the final manuscript and agree to be accountable for all aspects of the work.

\section{Ethics approval and consent to participate}

Not applicable.

\section{Patient consent for publication}

Not applicable.

\section{Competing interests}

The authors declare that they have no competing interests.

\section{References}

1. Siegel RL, Miller KD and Jemal A: Cancer Statistics, 2017. CA Cancer J Clin 67: 7-30, 2017.

2. Miller KD, Siegel RL, Lin CC, Mariotto AB, Kramer JL, Rowland JH, Stein KD, Alteri R and Jemal A: Cancer treatment and survivorship statistics, 2016. CA Cancer J Clin 66: 271-289, 2016.

3. Novello S, Barlesi F, Califano R, Cufer T, Ekman S, Levra MG, Kerr K, Popat S, Reck M, Senan S, et al: Metastatic non-small-cell lung cancer: ESMO clinical practice guidelines for diagnosis, treatment and follow-up. Ann Oncol 27 (Suppl 5): v1-v27, 2016.

4. Chen W, Zheng R, Baade PD, Zhang S, Zeng H, Bray F, Jemal A, Yu XQ and He J: Cancer statistics in China, 2015. CA Cancer J Clin 66: 115-132, 2016.

5. Milon L, Rousseau-Merck MF, Munier A, Erent M, Lascu I, Capeau J and Lacombe ML: nm23-H4, a new member of the family of human $\mathrm{nm} 23 /$ nucleoside diphosphate kinase genes localised on chromosome 16p13. Hum Genet 99: 550-557, 1997.
6. Schlattner U, Tokarska-Schlattner M, Epand RM, Boissan M, Lacombe ML, Klein-Seetharaman $J$ and Kagan VE: Mitochondrial NM23-H4/NDPK-D: A bifunctional nanoswitch for bioenergetics and lipid signaling. Naunyn Schmiedebergs Arch Pharmacol 388: 271-278, 2015.

7. Kagan VE, Jiang J, Huang Z, Tyurina YY, Desbourdes C, Cottet-Rousselle C, Dar HH, Verma M, Tyurin VA, Kapralov AA, et al: NDPK-D (NM23-H4)-mediated externalization of cardiolipin enables elimination of depolarized mitochondria by mitophagy. Cell Death Differ 23: 1140-1151, 2016.

8. Fujita Y, Fujiwara K, Zenitani S and Yamashita T: Acetylation of NDPK-D regulates its subcellular localization and cell survival. PLoS One 10: e0139616, 2015.

9. Xia SH, Wang J and Kang JX: Decreased n-6/n-3 fatty acid ratio reduces the invasive potential of human lung cancer cells by downregulation of cell adhesion/invasion-related genes. Carcinogenesis 26: 779-784, 2005.

10. Schlattner U, Tokarska-Schlattner M, Epand RM, Boissan M, Lacombe ML and Kagan VE: NME4/nucleoside diphosphate kinase $\mathrm{D}$ in cardiolipin signaling and mitophagy. Lab Invest 98: 228-232, 2018.

11. Amaravadi R, Kimmelman AC and White E: Recent insights into the function of autophagy in cancer. Genes Dev 30: 1913-1930, 2016.

12. Seifert M, Welter C, Mehraein Y and Seitz G: Expression of the $\mathrm{nm} 23$ homologues $\mathrm{nm} 23-\mathrm{H} 4, \mathrm{~nm} 23-\mathrm{H} 6$, and $\mathrm{nm} 23-\mathrm{H} 7$ in human gastric and colon cancer. J Pathol 205: 623-632, 2005.

13. Xu ZY, Chen JS and Shu YQ: Gene expression profile towards the prediction of patient survival of gastric cancer. Biomed Pharmacother 64: 133-139, 2010.

14. Hayer J,Engel M, Seifert M, Seitz G and Welter C: Overexpression of nm23-H4 RNA in colorectal and renal tumours. Anticancer Res 21: 2821-2825, 2001.

15. Patocs A, Zhang L, Xu Y, Weber F, Caldes T, Mutter GL, Platzer P and Eng C: Breast-cancer stromal cells with TP53 mutations and nodal metastases. N Engl J Med 357: 2543-2551, 2007.

16. Skotheim RI, Autio R, Lind GE, Kraggerud SM, Andrews PW, Monni O, Kallioniemi O and Lothe RA: Novel genomic aberrations in testicular germ cell tumors by array-CGH, and associated gene expression changes. Cell Oncol 28: 315-326, 2006.

17. Gaiser T, Thorns C, Merz H, Noack F, Feller AC and Lange K: Gene profiling in anaplastic large-cell lymphoma-derived cell lines with cDNA expression arrays. J Hematother Stem Cell Res 11: 423-428, 2002.

18. Guo JY and White E: Autophagy, metabolism, and cancer. Cold Spring Harb Symp Quant Biol 81: 73-78, 2016.

19. Milon L, Meyer P, Chiadmi M, Munier A, Johansson M, Karlsson A, Lascu I, Capeau J, Janin J and Lacombe ML: The human nm23-H4 gene product is a mitochondrial nucleoside diphosphate kinase. J Biol Chem 275: 14264-14272, 2000.

20. Livak KJ and Schmittgen TD: Analysis of relative gene expression data using real-time quantitative PCR and the 2(-Delta Delta C(T)) method. Methods 25: 402-408, 2001.

21. Postel EH: Multiple biochemical activities of NM23/NDP kinase in gene regulation. J Bioenerg Biomembr 35: 31-40, 2003.

22. Kowluru A, Tannous M and Chen HQ: Localization and characterization of the mitochondrial isoform of the nucleoside diphosphate kinase in the pancreatic beta cell: Evidence for its complexation with mitochondrial succinyl-CoA synthetase. Arch Biochem Biophys 398: 160-169, 2002.

23. Boissan M, Dabernat S, Peuchant E, Schlattner U, Lascu I and Lacombe ML: The mammalian Nm23/NDPK family: From metastasis control to cilia movement. Mol Cell Biochem 329: 51-62, 2009.

24. Janin J, Dumas C, Moréra S, Xu Y, Meyer P, Chiadmi M and Cherfils J: Three-dimensional structure of nucleoside diphosphate kinase. J Bioenerg Biomembr 32: 215-225, 2000.

25. Redmann M, Dodson M, Boyer-Guittaut M, Darley-Usmar V and Zhang J: Mitophagy mechanisms and role in human diseases. Int J Biochem Cell Biol 53: 127-133, 2014.

26. Gebert N, Joshi AS, Kutik S, Becker T, McKenzie M, Guan XL, Mooga VP, Stroud DA, Kulkarni G, Wenk MR, et al: Mitochondrial cardiolipin involved in outer-membrane protein biogenesis: Implications for Barth syndrome. Curr Biol 19: 2133-2139, 2009.

27. Porporato PE, Payen VL, Pérez-Escuredo J, De Saedeleer CJ, Danhier P, Copetti T, Dhup S, Tardy M, Vazeille T, Bouzin C, et al: A mitochondrial switch promotes tumor metastasis. Cell Rep 8: 754-766, 2014.

This work is licensed under a Creative Commons Attribution-NonCommercial-NoDerivatives 4.0 International (CC BY-NC-ND 4.0) License. 